\title{
Theranostics
}

2012; 2(3):283-294. doi: 10.7150/thno.3642

Review

\section{Biomedical Applications of Graphene}

\section{He Shen ${ }^{1}$, Liming Zhang ${ }^{1,2}$, Min Liu', and Zhijun Zhang ${ }^{1}{ }^{凶}$}

1. Division of Nanobiomedicine, Suzhou Institute of Nano-tech and Nano-bionics, Chinese Academy of Sciences, 398 Ruoshui Road, Suzhou, 215123, China;

2. Hunan Key Laboratory of Green Packaging and Application of Biological Nanotechnology, Hunan University of Technology, Zhuzhou 412008, China.

Corresponding author: Professor Zhijun Zhang, Division of Nanobiomedicine, Suzhou Institute of Nano-tech and Nano-bionics, Chinese Academy of Sciences, 398 Ruoshui Road, Suzhou, 215123, China. Tel: 86-512-62872556; Fax: 86-512-62603079; E-mail: zjzhang2007@sinano.ac.cn

(C) Ivyspring International Publisher. This is an open-access article distributed under the terms of the Creative Commons License (http:/ / creativecommons.org/ licenses/by-nc-nd/3.0/). Reproduction is permitted for personal, noncommercial use, provided that the article is in whole, unmodified, and properly cited.

Received: 2011.10.15; Accepted: 2011.11.16; Published: 2012.03.05

\begin{abstract}
Graphene exhibits unique 2-D structure and exceptional phyiscal and chemical properties that lead to many potential applications. Among various applications, biomedical applications of graphene have attracted ever-increasing interests over the last three years. In this review, we present an overview of current advances in applications of graphene in biomedicine with focus on drug delivery, cancer therapy and biological imaging, together with a brief discussion on the challenges and perspectives for future research in this field.
\end{abstract}

Key words: graphene, biomedical application, drug delivery, biosensing, bioimaging.

\section{Introduction}

Graphene, a novel two-dimensional nanomaterial composed of $\mathrm{sp}^{2}$-bonded carbon atoms, possesses a number of extraordinary electronic, optical, thermal and mechanical properties [1-5]. With the rapid deveolopment of synthesis and functionalization approches, graphene and its related derivatives have shown outstanding potentials in many fields, such as nanoelectronics [6], composite materials [7-11], energy technology (for examples, fuel cell, supercapacitor, hydrogen storage) [12-14], sensors [15], and catalysis [16], which have been summarised by several review articles [17-21].

Beyond the applications aforementioned, the biomedical application of graphene is a relative new area with significant potential. Since the seminal report on use of graphene oxide (GO) as an efficient nanocarrier for drug delivery by Dai et al. [22] in 2008, the first study on graphene for biomedical applications, a lot of interesting work has been carried out to explore the use of graphene for widespread biomedical applications, ranging from drug/gene delivery, biological sensing and imaging, antibacterial materials, to biocompatible scaffold for cell culture. The intensive research on the bioapplications of graphene and its derivatives is due to many fascinating properties, such as high specific surface area $\left(2630 \mathrm{~m}^{2} / \mathrm{g}\right)$, exceptional eletronic conductivity (mobility of charge carriers, $\left.200,000 \mathrm{~cm}^{2} \mathrm{~V}^{-1} \mathrm{~s}^{-1}\right)$, thermal conductivity $(\sim 5000 \mathrm{~W} / \mathrm{m} / \mathrm{K})$, mechanical strength (Young's modulus, $\sim 1100 \mathrm{Gpa}$ ) of graphene, and, intrinsic biocompatibility, low cost and scalable production, and facile biological/chemical functionalization of GO $[20,21]$.

The exciting achievements obtained in the last 3 years have been reviewed by Liu et al. [17,23], Li, Lin and coworkers [18], and Jiang [20]. Therefore we do not intend to give a systematic and detailed review on this topic, instead, we will give a brief overview of 
recent work that is updated and complementary to those described in the reviews mentioned above.

\section{Drug/Gene Delivery and Cancer Therapy}

\section{I Drug delivery}

GO, produced by vigorous oxidation of graphite by Hummers method [24], is an ideal nanocarrier for efficient drug and gene delivery. GO used for drug delivery is usually 1-3 layers (1-2 nm thick), with size ranging from a few nanometers to several hundred nanometers $[19,25,26]$. The unique structural features, such as large and planar $\mathrm{sp}^{2}$ hybridized carbon domain, high specific surface area $\left(2630 \mathrm{~m}^{2} / \mathrm{g}\right)$, and enriched oxygen-containing groups, render GO excellent biocompatibility, and physiological solubility and stability, and capability of loading of drugs or genes via chemical conjugation or physisorption approaches. Moreover, the reactive $\mathrm{COOH}$ and $\mathrm{OH}$ groups $\mathrm{GO}$ bears facilitate conjugation with various systems, such as polymers [27], biomolecules (biotargeting ligand [26], DNA [28], protein [29-31]), quantum dots [32], $\mathrm{Fe}_{3} \mathrm{O}_{4}$ nanoparticles [33], and others [34]), imparting GO with multi-functionalities and multi-modalities for diverse biological and medical applications.

Inspired by the ideas for carbon nanotubes-based drug delivery [23,35-39], Dai et al. explored for the first time nanoscale GO (NGO) as a novel and efficient nanocarrier for delivery of water insoluble aromatic anticancer drugs into cells [22]. In their approach, NGO was first conjugated with an amine-terminated six armed polyethylene glycol (PEG) molecule, followed by loading of a water insoluble anticancer drug, SN38 onto NGO surface by simple non-covalent adsorption via ח-ח stacking. The PEG-functionalized NGO loaded with SN38 exhibited high cytotoxicity for HCT-116 cells, 1000-fold more potent than CPT-11. In another work, the same group studied targeted delivery of chemical drugs into cells by using a Rituxan (CD20+ antibody) conjugated NGO-PEG [26] (Figure 1). This work further demonstrated that the drug release from the GO surface was $\mathrm{pH}$ dependent, suggesting the possibility of $\mathrm{pH}$-controlled drug release. The $\mathrm{pH}$-sensitive drug release behavior from many different GO-based drug delivery systems was also studied later by Chen et al. [40], Shi and colleagues [9], Misra et al. [41], and our group [42]. Apart from $\mathrm{pH}$-activated drug release, recently Pan and colleagues [43] developed a thermo-responsive drug delivery cargo, poly(N-isopropylacrylamide) grafted graphene sheets.

Combined use of multiple drugs is a widely adopted clinical practice in cancer therapy to overcome drug resistance of cancer cells [44,45]. However, few reports on nanomaterial-based drug delivery systems for controlled loading and delivery of multiple drugs can be found in the literature due to technical difficulties. To address this issue, our group [42] explored the feasibility of GO as a nanocarrier for controlled loading and targeted delivery of mixed chemical drugs. In our approach, folic acid and $\mathrm{SO}_{3} \mathrm{H}$ groups conjugated GO was co-loaded with two chemical drugs, doxorubicin (DOX) and camptothecin (CPT) via п-п stacking in a controlled way. We revealed that combined loading of the two drugs by GO with folic acid ligand exhibits specific targeting and much higher cytotoxicity to MCF-7 cells, human breast cancer cells with folic acid receptors, and more importantly, remarkably higher cytotoxicity than that loaded with only one drug.
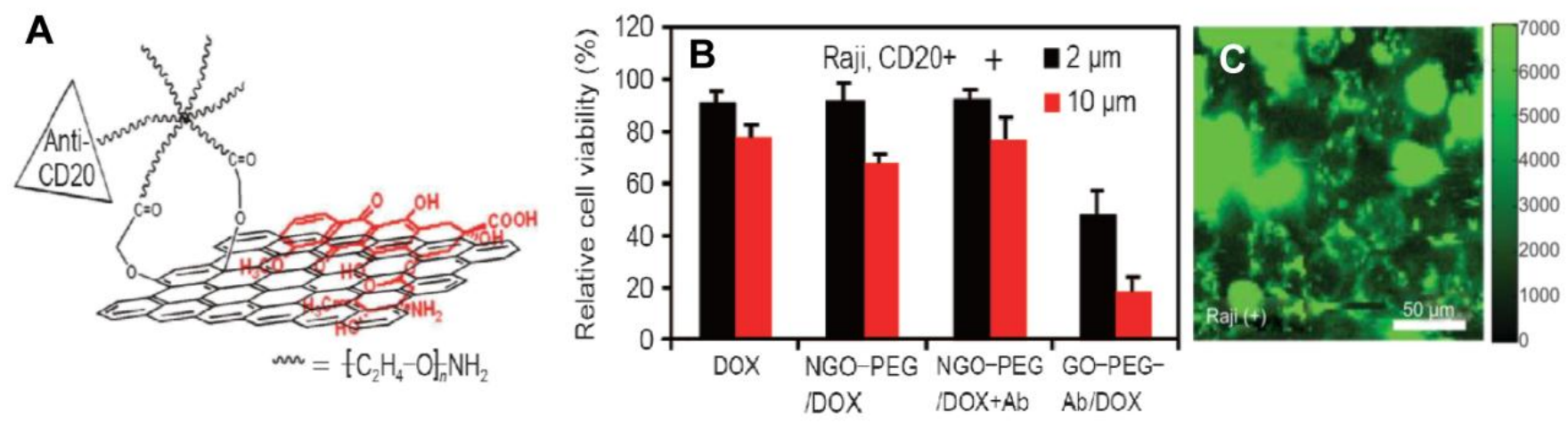

Figure I. A. Schematic illustration of DOX loading onto NGO-PEG conjugated with anti-CD20 antibody; B. In vitro cytotoxicity at different DOX concentration showing targeted delivery of DOX into specific cells. C. NIR fluorescence image of targeted cells treated with the NGO-PEG of Rituxan.[26] @ 2008. Reproduced with kind permission from Springer Science+Business Media and Tsinghua Press. 
The exploration of GO-based drug delivery expands from anticancer drugs to other drugs for non-cancer diseases treatment. Most recently, Rana et al. [46] reported the delivery of an anti-inflammatory drug, Ibuprofen, by using a chitosan-grafted GO. In this case, the loading rate of Ibuprofen on the GO sheet was determined to be $9.7 \%$. Furthermore, the work demonstrates that controlled drug release can be achieved by adjustment of $\mathrm{pH}$ value.

To enhance the anticancer effect, Yang and colleagues [47] designed and prepared a magnetic- and bio- dual targeting drug delivery cargo based on GO-Fe ${ }_{3} \mathrm{O}_{4}$ nanoparticle hybrid. The in vitro experiments indicated specific targeting of the multifunctional drug carriers by SK3 human breast cancer cells. Clearly, in vivo study is desired to demonstrate the performance of this external magnetic field-guided and bio-targeted drug delivery system.

\subsection{Gene delivery}

Gene therapy is a novel and promising approach to treat various diseases caused by genetic disorders, including cystic fibrosis, Parkinson's disease, and cancer [48]. Successful gene therapy requires a gene vector that protects DNA from nuclease degradation and facilitates cellular uptake of DNA with high transfection efficiency [49]. The major challenge facing the development of gene therapy is lack of efficient and safe gene vectors [50]. Recently Liu et al. [51] and our group [52] studied gene delivery using polyeth- ylenimine (PEI)-modified GO (PEI-GO), the procedure of which is shown in Figure 2A. GO conjugated with positively charged PEI allows condensation of plasmid DNA onto the surface of GO sheet through electrostatic interaction arising from the cationic polymer. Liu and colleagues [51] examined the transfection efficiency of GO-PEI-10K and GO-PEI-1.2K and compared with the unbound polymers PEI-10K and PEI-1.2K, respectively, as shown in Figure 2B. Their experiment indicates that grafting PEI to GO not only significantly lowered the cytotoxicity of the cationic polymer, but also improved the transfection efficiency of the polymer. We also found much higher luciferase expression in HeLa cells with PEI-GO/pGL-3 complexes $\left(2.75 \times 10^{9} \mathrm{RLU} / \mathrm{mg}\right.$ protein ) than that of PEI/pGL-3 complexes $\left(2.07 \times 10^{9}\right)$, and high DNA transfection efficiency by PEI-GO in HeLa cells even in the presence of $10 \%$ FBS [52]. This observation is somewhat surprising since PEI is widely used in gene transfection due to its high transfection efficiency, PEI grafted to other systems usually leads to inevitable decrease in transfection efficiency. And, serum protein can hinder cellular uptake, and therefore often reduces the transfection efficiency [52]. Much work has to be done to understand the phenomenon. All together, the findings by Liu and us suggest that PEI-GO is a promising candidate for efficient gene delivery.
A

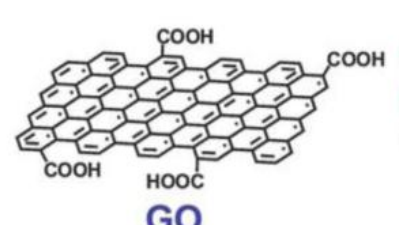

GO

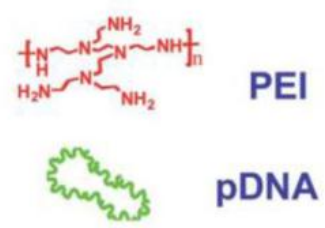

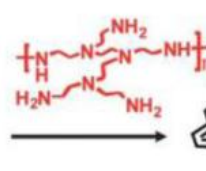

.

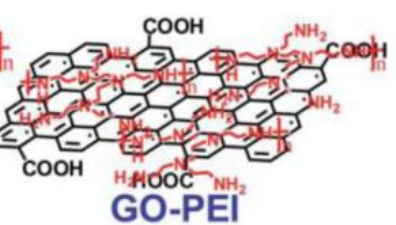

$\mathrm{GO}-\mathrm{PEI}$

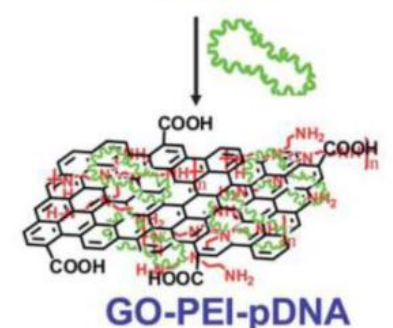

\section{B}

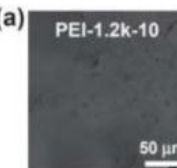

(b)
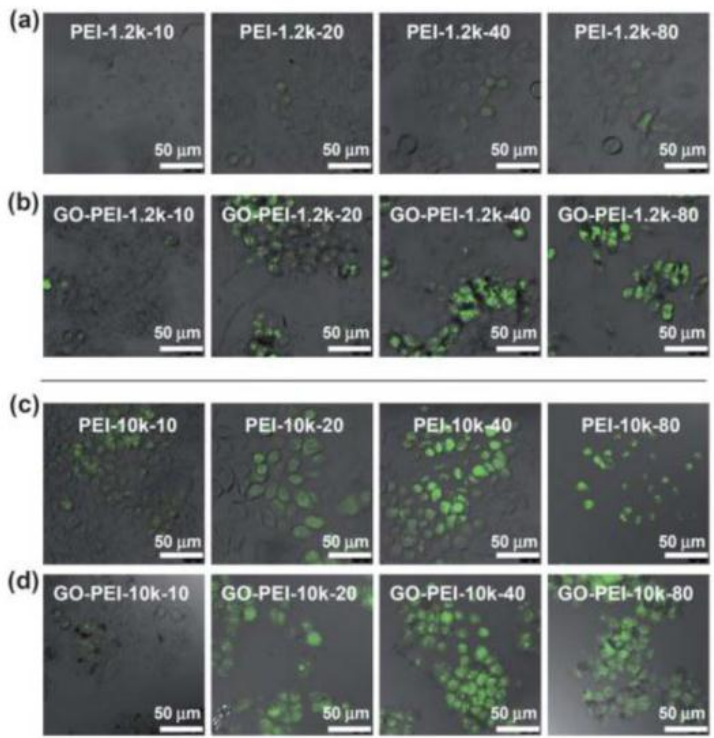

Figure 2. A. Schematic illustration of synthesizing GO-PEI-DNA complexes via electrostatic interactions. B. Confocal fluorescence images of EGFP transfected HeLa cells using PEI-I.2k (a), GO-PEI-I.2k (b), PEI-IOk (c), and GO-PEI-I0k (d) at different N/P ratios from IO to 80. [5I] Reproduced by permission of The Royal Society of Chemistry. 
Most recently a research group from Singapore [53] reported synthesis of chitosan-functionalized GO (GO-CS) sheets and their application for drug/gene delivery. This work shows that GO-CS sheets have a high drug payload, and the CPT-loaded GO-CS exhibits better cancer cell killing ability than the pure CPT. At the same time, GO-CS could condense plasmd DNA into stable nanoparticles, which show reasonable DNA transfection efficiency in HeLa cells at certain nitrogen/phosphate ratio. Further work on simultaneous loading and delivery of chemical drug and gene by the GO-CS nanocarrier for combined chemo- and gene- therapy is highly desired, to gain an enhanced therapeutic efficacy.

Our group studied sequential delivery of DOX

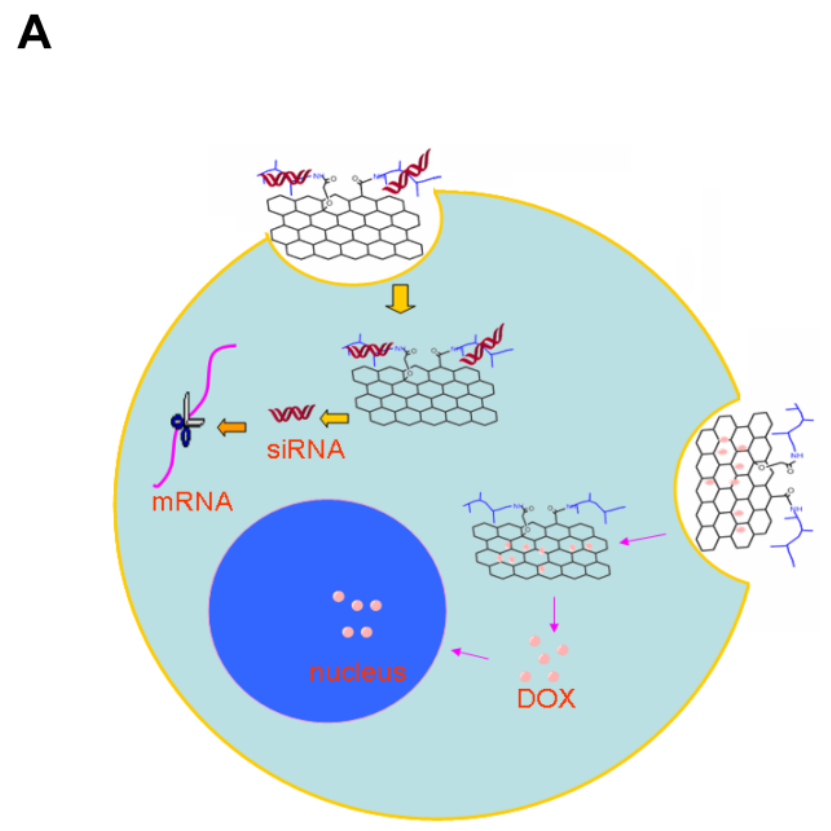

and Bcl-2 targeted siRNA to HeLa cells using a PEI-GO nanocarrier [54] (Figure 3). Briefly, HeLa cells were first incubated with the GO-PEI loaded with Bcl-2 targeted siRNA for $5 \mathrm{~h}$, and the incubation was continued for another $43 \mathrm{~h}$ after the fresh medium was replaced. The cells were then treated with DOX-loaded PEI-GO complexes for $24 \mathrm{~h}$. The cytotoxicity assay reveals that the PEI-GO/Bcl-2 targeted siRNA complexes dramatically enhanced the cytotoxicity of the PEI-GO/DOX, compared to the PEI-GO/scrambled siRNA complexes, as evidenced by remarkable decrease in $\mathrm{IC}_{50}$ of DOX, due to strong synergistic anti-cancer effect of the PEI-GO/DOX and PEI-GO/Bcl-2 targeted siRNA.

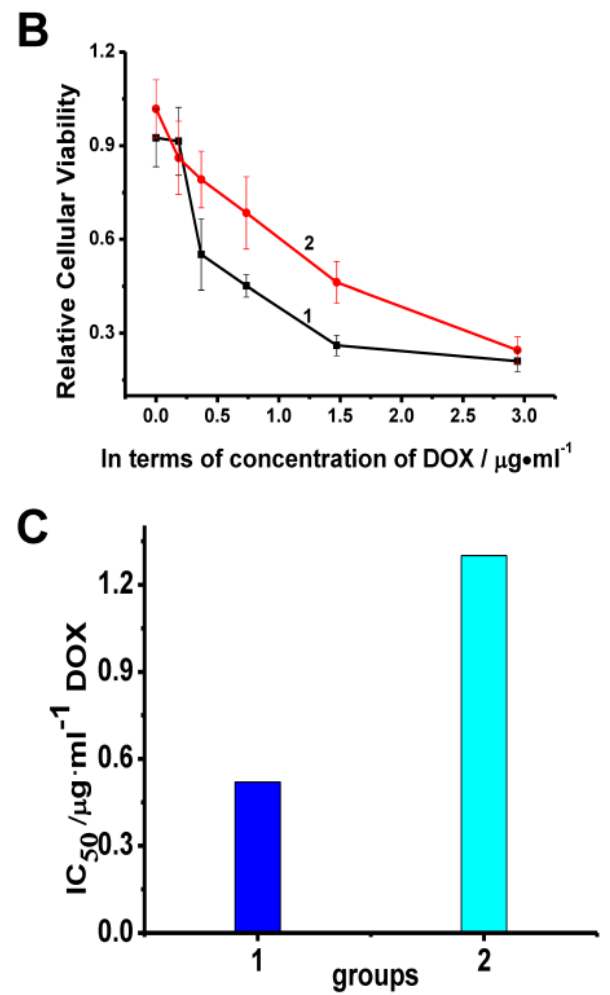

Figure 3. A. Scheme showing sequential delivery of siRNA and anticancer drugs by PEI-GO. B. Relative viability of HeLa cells treated with (I) PEI-GO/Bcl-2 targeted siRNA, and (2) PEI-GO/scrambled siRNA, followed by incubation with PEI-GO/DOX. C. IC 50 of DOX for the HeLa cells sequentially incubated with (I) PEI-GO/Bcl-2 targeted siRNA and PEI-GO/DOX, and (2) PEI-GO/scrambled siRNA and $\mathrm{PEI}-\mathrm{GO} / \mathrm{DOX}$. The result indicates that PEI-GO/Bcl-2-targeted siRNA complexes significantly enhanced the cytotoxicity of the PEI-GO/DOX. [54] Copyright Wiley-VCH Verlag GmbH \& Co. KGaA. Reproduced with permission.

\subsection{Cancer Therapy}

Much success has been made on exploration of graphene in drug delivery by in vitro test, as we discussed above. For clinical cancer and other disease treatment, the in vivo behavior of graphene loaded with drugs must be investigated. Liu and colleagues [55] for the first time studied in vivo tumor uptake and photothermal therapy with PEGylated GO using xenograft tumor mouse models. They observed very high tumor uptake of the PEG-modified GO due to highly efficient tumor passive targeting of GO caused by EPR effect. Moreover, under the low-power near-infrared (NIR) laser irradiation on the tumor, a highly efficient tumor destruction was achieved, taking the use of strong absorbance of GO in the NIR region (Figure 4). 

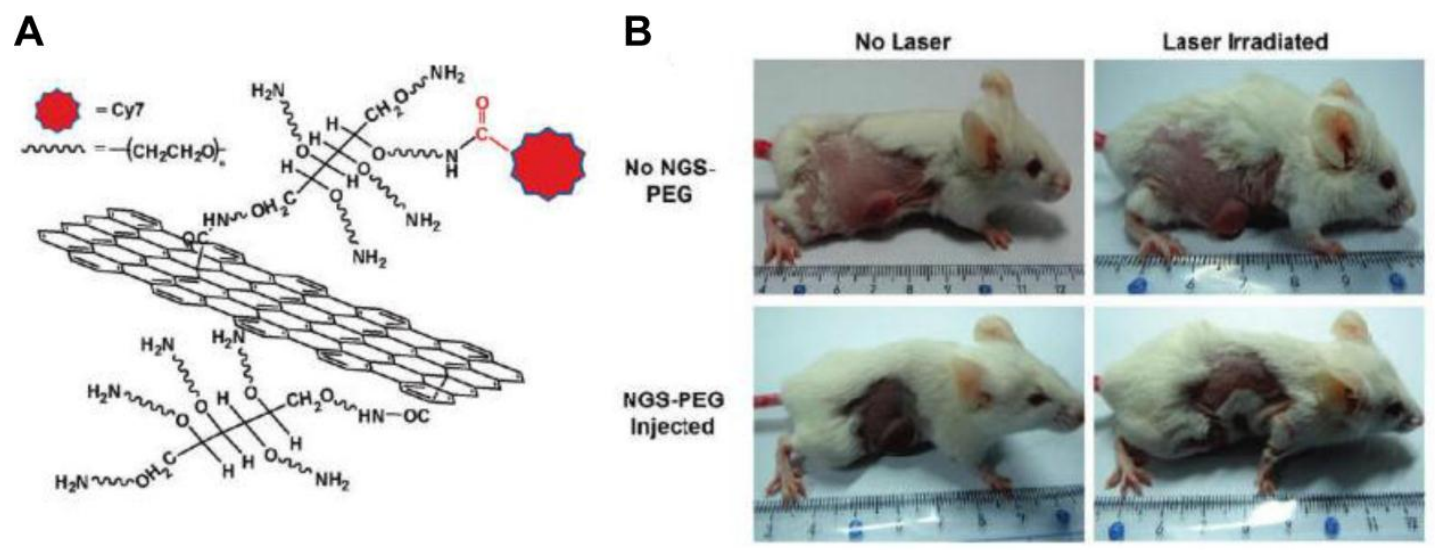

Figure 4. A. Schematic illustration of PEG functionalized NGS and labeled by Cy7. B. Photos of tumors on mice after various treatments indicated. The laser irradiated tumor on NGS injected mouse was completely destructed.[55] Reprinted with permission from ref.55. Copyright 2010 American Chemical Society.

Later, Markovic et al. [56] compared the photothermal anticancer activity of NIR-excited graphene and carbon nanotubes. Despite of its lower NIR-absorbing capacity, polyvinylpyrrolidone-coated graphene nanoparticles display higher photothermal responsiveness, and induce more photothermal cell death because of oxidative stress and mitochondrial depolarization and caspase activation leaking cytochrome $\mathrm{C}$ of human glioma cells in vitro than single-walled CNT [57].

Zhang and coworkers [58] developed a NGO-PEG-DOX for the anti-tumor effect in vitro and in vivo by combination of photothermal- and chemo-therapies. The experiment revealed that the combined chemo-photothermal therapy exhibited synergistic effect that leads to better cancer killing effect than chemotherapy or photothermal therapy alone.

Cui and his coworkers [59] reported application of folic acid and sulfonic acid conjugated GO loaded with porphyrin photosensitizers for targeting photodynamic therapy (PDT). PDT is a novel and efficient technique to treat diseases including cancers in clinical practice because of its attractive traits [60] with low toxicity and high stability under physiological conditions. In their experiment, GO was loaded with a Chlorin e6 photosensitizer with high efficiency via hydrophobic interactions and п-п stacking. Such system, significantly increases the accumulation of photosensitizers in tumor cells, leading to a remarkable concentration-depended photodynamic effect on cancer cells under irradiation.

Liu and colleagues [61] further studied photothermally assisted PDT using GO loaded with a photosensitizer, Chlorine e6, and demonstrated that such combined treatment yields remarkably improved cancer killing effect.

In summary, the structural features and facile chemical processing and modification of GO provide fascinating opportunities for loading and delivery of a variety of molecules, from chemical drugs, genes, and photosensitizers, and beyond, for cancer and other disease treatment. The research in this area so far is encouraging, but far from mature, more exciting accomplishments will be made, moving forward toward the eventual clinical use.

\section{Biosensing and Bioimaging}

\section{I Biosensing}

Graphene derivatives, including pristine graphene, GO, chemically reduced GO (rGO) [62] and doped graphene [63] have been intensively studied for their widespread applications in biosensing and detection of biomolecules such as thrombin [64], ATP [65], oligonucleotide [66], amino acid [67], and dopamine [68]. Several types of GO-based biosensors have been built, which include: (1) Making use of super efficient fluorescence quenching ability of graphene, some novel fluorescence resonance energy transfer (FRET) based biosensors have been developed [64,65,69]; (2) Based on the unique electronic property of graphene, FET type biosensors have been made [70]; (3) Controllable self-assembling of graphene-biomolecules allows to build highly ultrasensitive biosensors for detection of DNA and other molecules [66,71-73]; (4) As a matrix for detection of molecules, graphene-based nanoplatform for matrix-assisted laser desorption/ionization time-of-flight mass spectrometry (MALDI-TOF MS) has been reported [67,74]; and (5) GO-based novel biosensors via 
electrochemical principle have been constructed by taking use of its huge surface area, good electrical conductivity, and excellent capability of loading various biomolecules via chemical or physical interactions $[68,75]$. All of these have been thoroughly reviewed by $\mathrm{Li}$, Lin and colleagues [18], Jiang [20], and others $[17,19,21,76]$. Therefore we will not go into the details in this overview.

\subsection{Bioimaging}

Exploration of feasibilities of GO in biological imaging with optical and magnetic modalities has just started recently [19,26,33,77]. Dai et al. [26] for the first time examined cellular uptake of PEG-modified GO loaded with chemical drugs using intrinsic fluorescence of GO in the NIR region. Later, another group [78] studied gelatin-grafted rGO labeled with a fluorescence dye for cellular imaging and drug delivery.

Recently researchers have begun to prepare smaller GO (with a size of $10 \mathrm{~nm}$ or less), often referred to as graphene quantum dots (GQDs), from chemical oxidation of graphite [79-81] and "bottom up" approach [82]. These GQDs exhibit intrinsic fluorescence, and can be used for bioimaging purpose. Pan et al. [79] synthesized GQDs with blue emission color through hydrothermal cutting of GO. These as-obtained GQDs are weakly fluorescent (quantum yield less than $10 \%$ ), much lower than conventional organic dyes and II-VI type QDs, which hampers their practical bioimaging applications. To overcome this problem, Eda et al. [80] treated the chemically produced GQDs by a brief exposure to hydrazine vapor and observed significantly improved fluorescence. Surface functionalization of GQD with alkylamine also gives rise to dramatically enhanced fluorescence [81]. Most recently, Zhu et al. [83] (Figure 5.) and our group [84] explored the possibility of GQDs for cellular imaging. Compared with CdSe and other II-VI type QDs, GQDs show excellent biocompatibility, physiological solubility, and low cytotoxicity, and can be used directly for intracellular imaging without the necessity for further surface processing or functionalization. In addition, GQDs possess unique optical properties such as $\mathrm{pH}$ dependent and upconversion fluorescence behaviors [85]. The upconversion fluorescence property of GQDs allows them to be excited at NIR region, making both in vitro and in vivo bio-detection and imaging efficient, safe, and without interference from auto-fluorescence from the cells, organs, or tissues in this region [86].
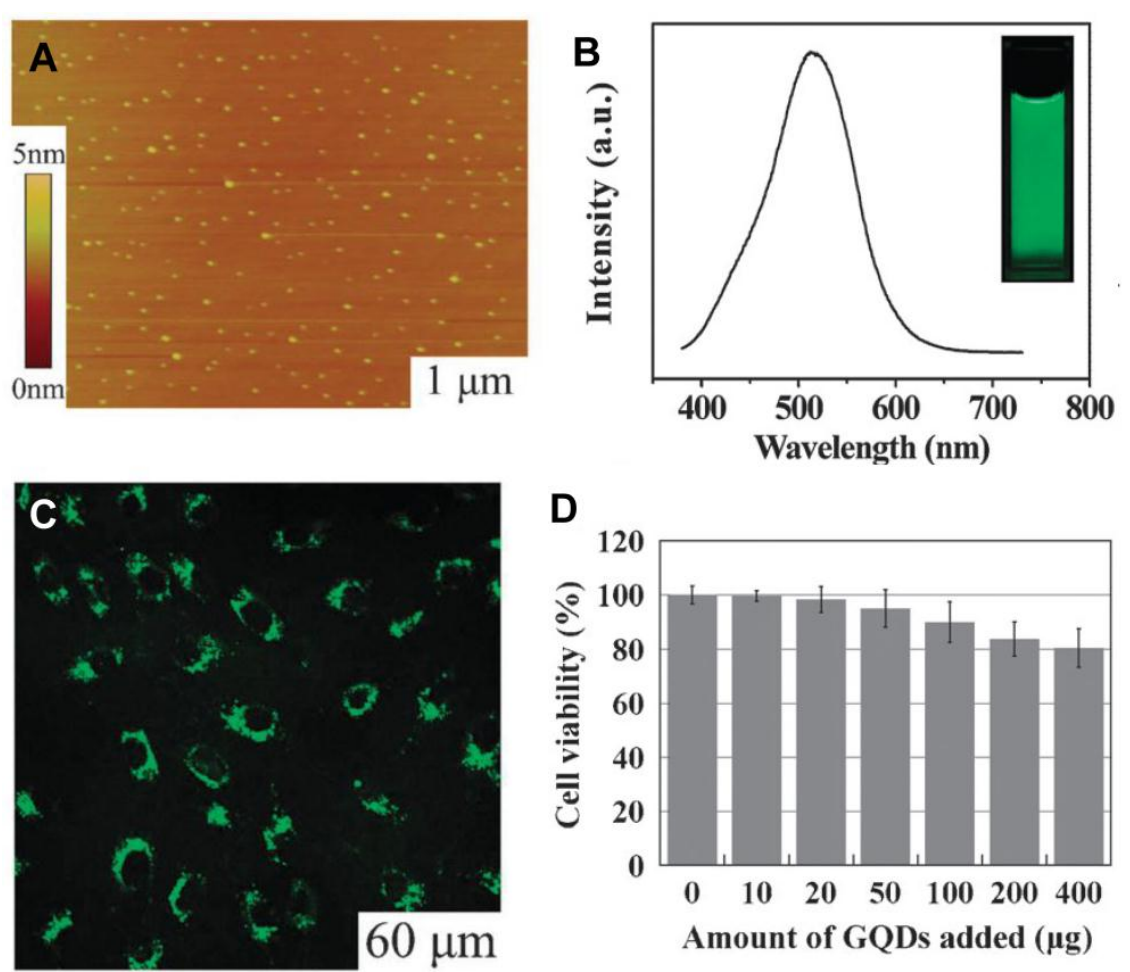

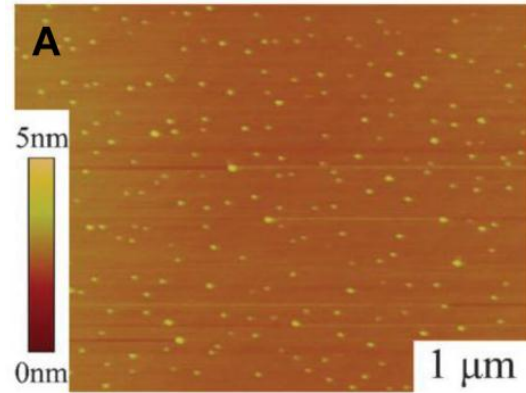

D

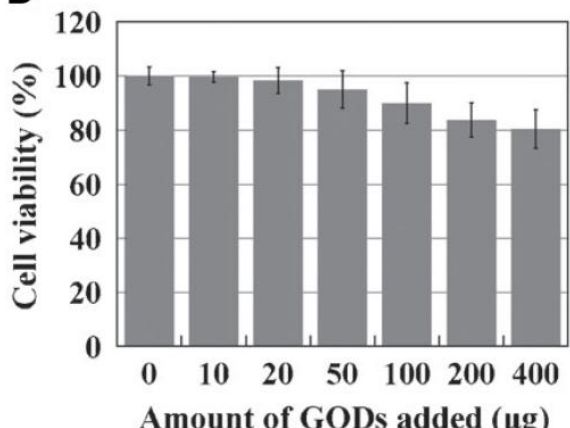

Figure 5. A. AFM image of the GQDs; B. Fluorescence spectra of aqueous solution of GQDs excited at $375 \mathrm{~nm}$. Inset: aqueous solution of GQDs under UV light; C. cellular imaging of GQDs imaged under $405 \mathrm{~nm}$; D. Cytoxicity of GQDs. [83] Reproduced by permission of The Royal Society of Chemistry. 
Like fluorescence imaging technique, magnetic resonance imaging (MRI) is widely used in clinical practice $[87,88]$. Recently our group [33] reported the synthesis of composites of dextran-coated $\mathrm{Fe}_{3} \mathrm{O}_{4}$ nanoparticles (NPs) and GO $\left(\mathrm{Fe}_{3} \mathrm{O}_{4}\right.$-GO) as $T_{2}$-weighted contrast agent for efficient cellular MRI. The $\mathrm{Fe}_{3} \mathrm{O}_{4}$-GO composites we prepared possess good physiological stability and low cytotoxicity. Compared with the isolated $\mathrm{Fe}_{3} \mathrm{O}_{4} \mathrm{NPs}$, the $\mathrm{Fe}_{3} \mathrm{O}_{4}$-GO composites exhibit significantly enhanced cellular MRI, able to detect cells at iron concentration of $5 \mu \mathrm{g}$ $\mathrm{mL}^{-1}$ with cell density of $2 \times 10^{5}$ cells $\mathrm{mL}^{-1}$, and at the iron concentration of $20 \mu \mathrm{g} \mathrm{mL}^{-1}$ with cell density of 1000 cells $\mathrm{mL}^{-1}$.
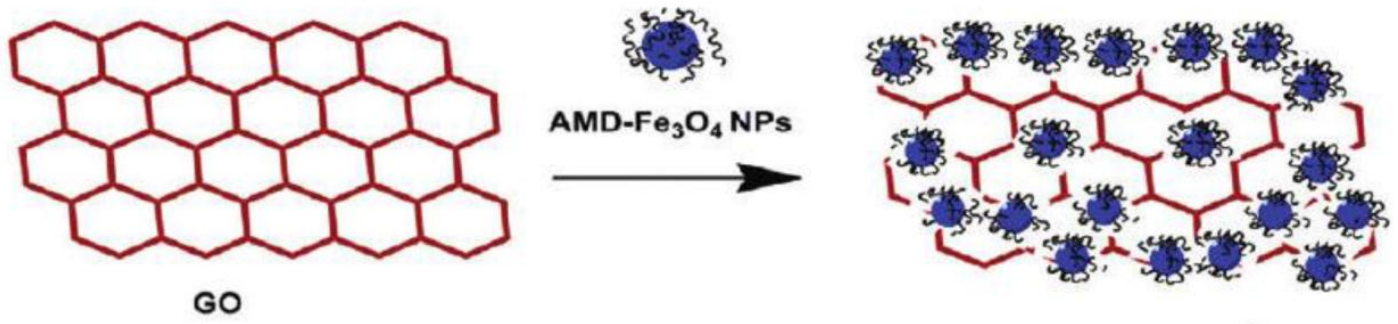

(a)
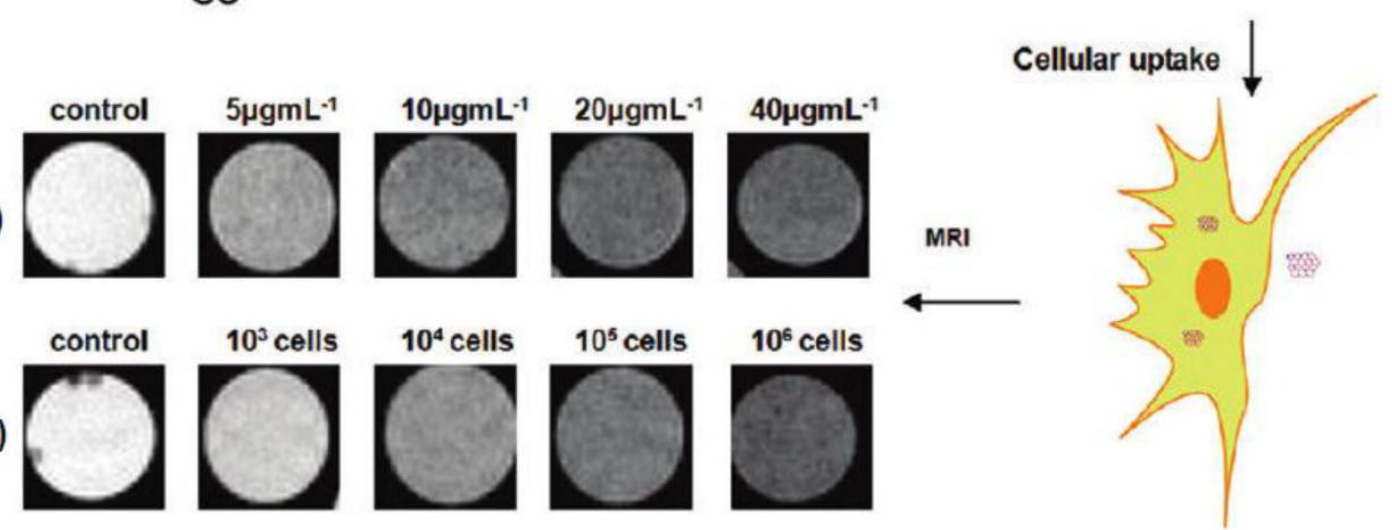

Figure 6. Schematic illustration of preparation of the $\mathrm{Fe}_{3} \mathrm{O}_{4}-\mathrm{GO}$ composites and $\mathrm{T}_{2}$ weighted cellurlar MR images: (a) $\mathrm{HeLa}$ cells $\left(2 \times 10^{5}\right.$ cells $\left.\mathrm{mL}^{-1}\right)$ incubated with the $\mathrm{Fe}_{3} \mathrm{O}_{4}-\mathrm{GO}$ composites at different concentrations. (b) $\mathrm{Fe}_{3} \mathrm{O}_{4}-\mathrm{GO}$ composites $(20 \mu \mathrm{g} \mathrm{Fe} \mathrm{mL-1)} \mathrm{incubated}$ with HeLa cells at different cell density which $\mathrm{Fe}_{3} \mathrm{O}_{4}$ NPs formed aggregations on the $\mathrm{GO}$ sheets, resulting in a considerable enhanced $\mathrm{T}_{2}$ relaxivity. [33] Reprinted with permission from ref.33. Copyright 20II American Chemical Society.

\section{Other applications}

\section{I GO-based antibacterial materials.}

Fan et al. [89] prepared macroscopic freestanding GO and rGO paper from their suspension by vacuum filtration technique, and found that these papers exhibit strong antibacterial effect. Considering the scalability and low cost of the graphene-based antibacterial paper, this work opens new opportunities for the use of GO in environmental and clinical applications.

Later Akhavan et al. [90] investigated anti-bacterial effect of graphene nanosheets in the form of nanowalls deposited on stainless steel substrates for both Gram-positive and Gram-negative models of bacteria. It is interesting that the Gram-negative Escherichia coli bacteria with an outer membrane were more resistant to the cell membrane damage caused by the nanowalls than the Gram-positive Staphylococcus aureus lacking the outer membrane. Moreover, the rGO nanowalls were more toxic to the bacteria than the GO nanowalls. The better antibacterial activity of the rGO nanowalls, according to the researchers, is due to the better charge transfer between the bacteria and the more sharpened edges of the rGO nanowalls, during the contact interaction.

Liu et al. [91] further probed the mechanism of antibacterial effect of 4 types of graphene derivatives, graphite (Gt), graphite oxide (GtO), GO, and rGO. They found that the antibacterial activities decrease in the order of GO, rGO, Gt, and GtO. They assign the antibacterial effect to both membrane and oxidative stress, and then proposed a three-step mechanism for the antibacterial effect, similar to that of carbon nanotube. This work is important for us to understand the GO-bacteria interaction and to guide development of better graphene-based antibacterial materials.

\subsection{GO-based scaffold for cell culture.}

Min group studied the behavior of NIH-3T3 fi- 
broblasts as a model of mammalian cells growing on a supported film of GO [92]. Their work suggests that GO film induces no significantly harmful effect on the mammalian cells with respect to adhesion, and exhibits remarkably high gene transfection efficiency, $250 \%$ that of cells grown on a cover glass substrate. The result indicated the potential application of GO as a surface coating material for implant.

A graphene/chitosan film produced by solution casting method has also been investigated as scaffold materials in tissue engineering [8]. The work indicates that the graphene-based film does not hamper the proliferation of human mesenchymal stem cells
(hMSCs). Instead, it accelerates their specific differentiation into bone cells in a controlled manner through the use of growth factors and osteogenic inducers, suggesting the potential use for proliferation and transplantation of stem cells and their specific differentiation into muscles, bones, and cartilages for bone regeneration therapy [93] (Figure 7). Another study [94] revealed that chemical vapor deposition (CVD) grown graphene substrate is biocompatible for human osteoblasts as well as for hMSCs with higher proliferation of cell, compared to $\mathrm{SiO}_{2}$ substrate, and stimulates the growth and differentiation of cells.
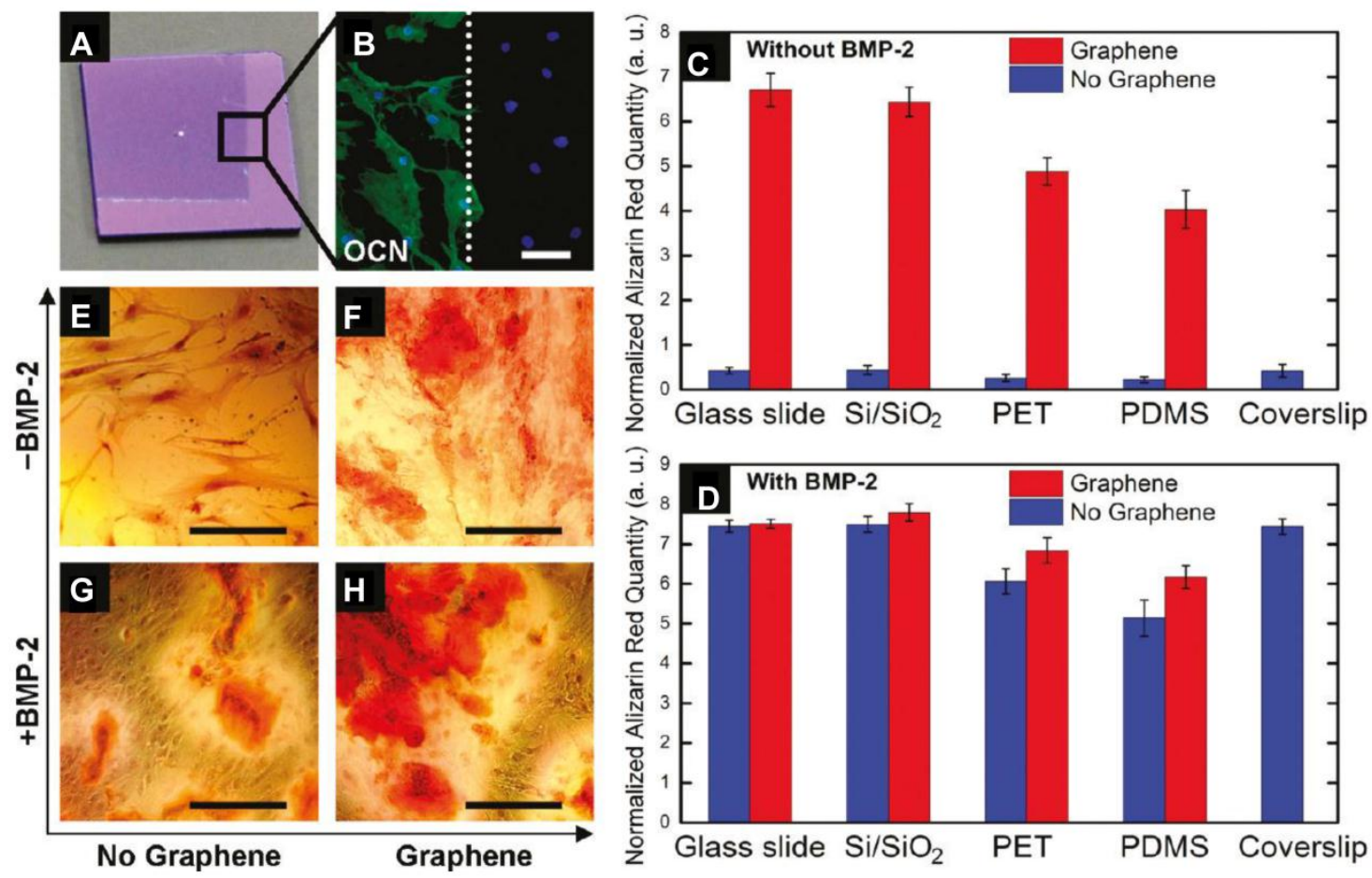

Figure 7. Graphene substrate for osteogenic differentiation. A. Optical image of graphene-coated Si/SiO2 chip, showing the graphene boundary. B. Osteocalcin (OCN) marker showing bone cell formation on the same chip only on the graphene-coated area; C-D. Alizarin red quantification deriving from hMSCs grown for 15 days on substrates with/without graphene as well as with/ without BMP-2. E-H. PET substrate stained with alizarin red showing calcium deposits due to osteogenesis. [93] Reprinted with permission from ref.93. Copyright 201 I American Chemical Society.

Inspired by the work mentioned above, Tang and Cheng et al [95] investigated CVD-grown graphene film as a substrate of neurites, one of the key structures for neural functions during the development in a mouse hippocampal culture model. Their results indicate that the neurite numbers average length on graphene film were significantly enhanced during 2-7 days after cell seeding compared with tissue culture polystyrene (TCPS) substrates. Moreover,
GAP-43 expression was greatly enhanced in graphene group compared to TCPS group, due likely to the boost of neurite sprouting and outgrowth. This study suggests the potential application of pristine graphene as a novel material for neural interfacing.

The interesting yet preliminary work described above suggests the potential applications of graphene as antibacterial materials and scaffold materials for cell culture. 


\section{Conclusions and perspectives}

As in many other fields, the research on biomedical applications of graphene has seen dramatic progress, and is expanding rapidly, yet mostly in its initial stage. The advances made in this area so far are exciting and encouraging, the challenges we face, however, are also very huge and must be overcome. One of such challenges is thorough and profound understanding of graphene-cell (or tissue, organ) interactions, especially the cellular uptake mechanism. Such knowledge certainly facilitates development of more efficient graphene or GO-based nanoplatform for drug delivery, biosensing and other applications, which, however, is still lacking at the moment. The toxicity of graphene and GO, at in vitro and in vivo level, is another major concern. At the current stage there are only a few publications in this regard [96-102]. The preliminary results indicate that the physicochemical properties such as flat shape and charges, are closely related to the cytotoxicity, and affect in vivo biodistribution and fate of GO [17]. The mechanisms of the in vitro biotoxicity caused by graphene are related to oxidative stress and damage of cell membrane. Clearly, a systematic study is highly desired to address the safety concerns before practical applications of graphene in biomedicine.

The current research on delivery of drugs, genes, we expect, will extend to that of proteins, growth factors, and other biomolecules, either single or combined use of the aforementioned biomolecules, for cancer and other disease therapies. Given its structural features and exceptional physicochemical properties, design and construction of GO-based theranostic platform with multifunctionalities and multimodalities is a new direction to pursue. Definitely, these goals can only be reached by the joint efforts from chemistry, biomedicine, materials sciences, and nanotechnology. To this end, development of suitable chemical synthesis and functionalization approaches for precise control over size, size distribution, morphology, structural defects, and oxygen-containing groups of GO is urgently needed, as this is closely correlated to the performance of the GO-based nanomaterials for biomedical applications, and the safety issues as well.

For GO-based biosensing based on FRET principle, tuning electronic property of GO by controllable modification and reduction of originally prepared $\mathrm{GO}$, and development of techniques to integrate $\mathrm{GO}$ into practical devices having high sensitivity, selectivity with acceptable reproducibility, reliability and low cost remain a big challenge. As for GO-based bioimaging, although the GQDs have distinct ad- vantages over II-IV QDs in their intrinsic biocompatibility, safety and facile functionalization, the weak fluorescence intensity (with quantum yield about $10 \%$ ) and broad emission band (with bandwidth beyond $100 \mathrm{~nm}$ ) are certainly major obstacles for their use in biodetection and labeling, so more efforts must be paid to prepare GQDs with a good control of size and size distribution, the surface defects, and their functionalization, to improve fluorescence quantum yield and other important properties.

The research on graphene/GO-based scaffold materials for cell culture is a relatively new direction that deserves special attention. The study in this field so far demonstrated that graphene and GO are able to accelerate the growth, differentiation, and proliferation of stem cells, and therefore hold great promise in tissue engineering, regenerative medicine, and other biomedical fields.

We have selectively reviewed the surprisingly rapid and exciting advances in the biomedical applications of graphene with focus on drug delivery and bioimaging, made in the last few years. This trend, we believe, will continue and even speed up in the years to come.

\section{Acknowledgement}

This work was supported by NSFC (No. 21073224), 973 Project (No. 2010 CB933504), and Innovation Project CAS (KJCX2. YW. M12).

\section{Conflict of Interest}

The authors have declared that no conflict of interest exists.

\section{References}

1. Novoselov KS, Geim AK, Morozov SV, et al. Electric field effect in atomically thin carbon films. Science. 2004; 306(5696):666-669.

2. Zhang K, Zhang LL, Zhao XS, et al. Graphene-polyaniline nanofiber composites as supercapacitor electrodes. Chem Mater. 2010; 22(4): 1392-1401.

3. Rao CNR, Sood AK, Subrahmanyam KS, et al. Graphene: The new two-dimensional nanomaterial. Angew Chem Int Ed. 2009; 48(42): 7752-7777.

4. Balandin AA, Ghosh S, Bao WZ, et al. Superior thermal conductivity of single-layer graphene. Nano Lett. 2008; 8(3): 902-907.

5. Latil S, Henrard L. Charge carriers in few-layer graphene gilms. Phys Rev Lett. 2006; 97(3) : 036803-6.

6. Xuan $\mathrm{Y}, \mathrm{Wu} \mathrm{YQ}$, Shen $\mathrm{T}$, et al. Atomic-layer-deposited nanostructures for graphene-based nanoelectronics. Appl Phys Lett. 2008; 92(1) : 013101-3.

7. Yang XM, Tu YF, Li L, et al. Well-dispersed chitosan/graphene oxide nanocomposites. ACS Appl Mater. Interfaces. 2010; 2 (6):1707-1713.

8. Fan HL, Wang LL, Zhao KK, et al. Fabrication, mechanical properties, and biocompatibility of graphene-reinforced chitosan composites. Biomacromolecules. 2010; 11(9): 2345-2351. 
9. Bai $\mathrm{H}, \mathrm{Li} \mathrm{C}$, Wang $\mathrm{XL}$, et al. A pH-sensitive graphene oxide composite hydrogel. Chem Commun. 2010; 46(14): 2376-2378.

10. Sun ST, Wu PY. A one-step strategy for thermal- and pH-responsive graphene oxide interpenetrating polymer hydrogel networks. J Mater Chem. 2011; 21(12): 4095-4097.

11. Fang F, Long J, Zhao WF, et al. pH-Responsive chitosan-mediated graphene dispersions. Langmuir. 2010; 26(22): 16771-16774.

12. Liu C, Alwarappan S, Chen ZF, et al. Membraneless enzymatic biofuel cells based on graphene nanosheets. Biosens Bioelectron. 2010; 25: 1829-1833.

13. Stoller MD, Park S, Zhu YW, et al. Graphene-based ultracapacitors. Nano Lett.2008; 8(10): 3498-3502.

14. Wang L, Lee $\mathrm{K}$, Sun YY, et al. Graphene oxide as an ideal substrate for hydrogen storage. ACS Nano. 2009; 3(10): 2995-3000.

15. Lu CH, Yang $\mathrm{HH}$, Zhu $\mathrm{CL}$, et al. A graphene platform for sensing biomolecules. Angew Chem Int Ed. 2009; 121(26): 4879-4881.

16. Qu LT, Liu Y, Baek JB, et al. Nitrogen-doped graphene as efficient metal-free electrocatalyst for oxygen reduction in fuel cells. ACS Nano. 2010; 4(3):1321-1326.

17. Feng LZ, Liu Z. Graphene in biomedicine: opportunities and challenges. Nanomedicine.2011; 6(2): 317-324.

18. Wang Y, Li ZH, Wang J, et al. Graphene and graphene oxide: biofunctionalization and applications in biotechnology. Trends Biotechnol. 2011; 29(5): 5205-212.

19. Loh KP, Bao QL, Eda G, et al. Graphene oxide as a chemically tunable platform for optical applications. Nat chem. 2010; 2 : 1015-1024.

20. Jiang HJ. Chemical preparation of graphene-based nanomaterials and their applications in chemical and biological sensors. Small. 2011; 7(17): 2413-2427.

21. Guo SJ, Dong SJ. Graphene nanosheet: synthesis, molecular engineering, thin film, hybrids, and energy and analytical applications. Chem Soc Rev. 2011; 40(5): 2644-2672.

22. Liu Z, Robinson JT, Sun XM, et al. PEGylated nanographene oxide for delivery of water-insoluble cancer drugs. J Am Chem Soc. 2008; 130(33): 10876-10877.

23. Liu Z, Robinson JT, Tabakman SM, et al. Carbon materials for drug delivery \& cancer therapy. Materials Today. 2011; 14(7-8): 316-323.

24. Hummers WS, Offeman RE. Preparation of Graphitic Oxide. J Am Chem Soc. 1958; 80: 1339-1339.

25. Kovtyukhova NI, Ollivier PJ, Martin BR, et al. Layer-by-layer assembly of ultrathin composite films from micron-sized graphite oxide sheets and polycations. Chem Mater. 1999; 11: 771-778.

26. Sun XM, Liu Z, Welsher $K$, et al. Nano-graphene oxide for cellular imaging and drug delivery. Nano Res. 2008; 1(3): 203-212.

27. Shan CS, Yang HF, Han DX, et al. Water-soluble graphene covalently functionalized by biocompatible poly-L-lysine. Langmuir. 2009; 25(20): 12030-12033.

28. Lei HZ, Mi LJ, Zhou XJ, et al. Adsorption of double-stranded DNA to graphene oxide preventing enzymatic digestion. Nanoscale. 2011; 3: 3888-3892.

29. Zhang JL, Zhang F, Yang HJ, et al. Graphene oxide as a matrix for enzyme immobilization. Langmuir. 2010; 26(9): 6083-6085.

30. Zhang F, Zheng B, Zhang JL, et al. Horseradish peroxidase immobilized on graphene oxide: physical properties and applications in phenolic compound removal. Phys Chem C. 2010; 114(18): 8469-8473.

31. Lee DY, Khatun Z, Lee JH, et al. Blood compatible graphene/heparin conjugate through noncovalent chemistry. Biomacromolecules. 2011; 12(2): 336-341.
32. Dong HF, Gao WC, Yan F, et al. Fluorescence resonance energy transfer between quantum dots and graphene oxide for sensing biomolecules. Anal Chem. 2010; 82(13): 5511-5517.

33. Chen WH, Yi PW, Zhang Y, et al. Composites of aminodextran-coated $\mathrm{Fe} 3 \mathrm{O} 4$ nanoparticles and graphene oxide for cellular magnetic resonance imaging. ACS Appl Mater Interfaces. 2011; 3(10): 4085-4091.

34. Shen JF, Shi M, Li N, et al. Facile synthesis and application of Ag-chemically converted graphene nanocomposite. Nano Res. 2010; 3(5): 339-349.

35. Dai HJ. Carbon nanotubes: opportunities and challenges. Surface Sci. 2002; 500: 218-241.

36. Liu Z, Tabakman S, Welsher $\mathrm{K}$, et al. Carbon nanotubes in biology and medicine: In vitro and in vivo detection, imaging and drug delivery. Nano Res. 2009; 2(2): 85-120.

37. Bianco A, Kostarelos K, Partidos CD, et al. Biomedical applications of functionalised carbon nanotubes. Chem Commun. 2005; 5: 571-577.

38. Bianco A, Kostarelos K, Prato M. Applications of carbon nanotubes in drug delivery. Curr Opin Chem Biol. 2005; 9:674-679.

39. Lu FS, Gu LR, Meziani MJ, et al. Advances in bioapplications of carbon nanotubes. Adv Mater. 2009; 21(2): 139-152.

40. Yang XY, Zhang XY, Liu ZF, et al. High-efficiency loading and controlled release of doxorubicin hydrochloride on graphene oxide. J Phys Chem C. 2008; 112(45): 17554-17558

41. Depan D, Shah J, Misra RDK. Controlled release of drug from folate-decorated and graphene mediated drug delivery system: Synthesis, loading efficiency, and drug release response. Mater Sci Eng C. 2011; 31(7): 1305-1312.

42. Zhang LM, Xia JG, Zhao QH, et al. Functional graphene oxide as a nanocarrier for controlled loading and targeted delivery of mixed anticancer drugs. Small. 2010; 6(4): 537-544.

43. Pan YZ, Bao HQ, Sahoo NG, et al. Water-soluble poly(N-isopropylacrylamide) -graphene sheets synthesized via click chemistry for drug delivery. Adv Funct Mater. 2011; 21(14): 2754-2763.

44. Andersson M, Lindegaard Madsen E, Overgaard M, et al. Doxorubicin versus methotrexate both combined with cyclophosphamide, 5-fluorouracil and tamoxifen in postmenopausal patients with advanced breast cancer-a randomised study with more than 10 years follow-up from the danish breast cancer cooperative group. Eur J Cancer. 1999; 35(1): 39-46.

45. Gavrilov V, Steiner M, Shany S. The combined treatment of 1,25-dihydroxyvitamin D3 and a non-steroid anti-inflammatory drug is highly effective in suppressing prostate cancer cell line (LNCaP) growth. Anticancer Res. 2005; 25(5): 3425-3429.

46. Rana VK, Choi MC, Kong JY, et al. Synthesis and drug-delivery behavior of chitosan-functionalized graphene oxide hybrid nanosheets. Macromol Mater Eng. 2011; 296(2): 131-140.

47. Yang XY, Wang YS, Huang X, et al. Multi-functionalized graphene oxide based anticancer drug-carrier with dual-targeting function and $\mathrm{pH}$-sensitivity. J Mater Chem. 2011; 21(10): 3448-3454.

48. Yang ZR, Wang HF, Zhao J, et al. Recent developments in the use of adenoviruses and immunotoxins in cancer gene therapy. Cancer Gene Ther. 2007; 14: 599-615.

49. Naldini L, Blömer U, Gallay P, et al. In Vivo gene delivery and stable transduction of nondividing cells by a lentiviral vector. Science. 1996; 272: 263-267.

50. Mintzer MA and Simanek EE. Nonviral vectors for gene delivery. Chem Rev. 2009; 109(2): 259-302.

51. Feng LZ, Zhang S, Liu Z. Graphene based gene transfection. Nanoscale. 2011; 3: 1252-1257.

52. Chen B, Liu M, Zhang LM, et al. Polyethylenimine-functionalized graphene oxide as an efficient gene delivery vector. J Mater Chem. 2011; 21: 7736-7741. 
53. Bao HQ, Pan YZ, Ping Y, et al. Chitosan-functionalized graphene oxide as a nanocarrier for drug and gene delivery. Small. 2011; 7(11): 1569-1578.

54. Zhang LM, Lu ZX, Zhao QH, et al. Enhanced chemotherapy efficacy by sequential delivery of siRNA and anticancer drugs using PEI-grafted graphene oxide. Small. 2011; 7(4): 460-464.

55. Yang K, Zhang S, Zhang GX, et al. Graphene in mice: ultrahigh in vivo tumor uptake and efficient photothermal therapy. Nano Lett. 2010; 10(9): 3318-3323.

56. Markovic ZM, Harhaji-Trajkovic LM, Todorovic-Markovic BM, et al. In vitro comparison of the photothermal anticancer activity of graphene nanoparticles and carbon nanotubes. Biomaterials. 2011; 32 : 1121-1129.

57. Kam NWS, O'Connell M, Wisdom JA, et al. Carbon nanotubes as multifunctional biological transporters and near-infrared agents for selective cancer cell destruction. Proc Nat Acad Sci. 2005; 102(33): 11600-11605.

58. Zhang W, Guo ZY, Huang DQ, et al. Synergistic effect of chemo-photothermal therapy using PEGylated graphene oxide. Biomaterials. 2011; 32(33): 8555-8561.

59. Huang $\mathrm{P}, \mathrm{Xu} \mathrm{C}$, Lin J, et al. Folic acid-conjugated graphene oxide loaded with photosensitizers for targeting photodynamic therapy. Theranostics. 2011; 1: 240-250.

60. Huang Z, Xu HP, Meyers AD, et al. Photodynamic therapy for treatment of solid tumors-Potential and technical challenges. Technol Cancer Res T. 2008; 7(4): 309-20.

61. Tian B, Wang C, Zhang S, et al. Photothermally enhanced photodynamic therapy delivered by nano-graphene oxide. ACS Nano, 2011; 5(9): 7000-7009.

62. Tang LH, Wang Y, Li YM, et al. Preparation, structure and electrochemical properties of graphene modified electrode. Adv Funct Mater. 2009; 19(17): 2782-2789.

63. Wang Y, Shao YY, Matson DW, et al. Nitrogen-doped graphene and its application in electrochemical biosensing. ACS Nano. 2010; 4(4): 1790-1798.

64. Chang HX, Tang LH, Wang Y, et al. Graphene fluorescence resonance energy transfer aptasensor for the thrombin detection. Anal Chem. 2010; 82(6): 2341-2346.

65. Wang Y, Li ZH, Hu DH, et al. Aptamer/graphene oxide nanocomplex for in situ molecular probing in living cells. J Am Chem Soc. 2010; 132(27): 9274-9276.

66. Tang LH, Wang Y, Liu Y, et al. DNA-directed self-assembly of graphene oxide with applications to ultrasensitive oligonucleotide assay. ACS Nano. 2011; 5(5): 3817-3822.

67. Dong XL, Cheng JS, Li JH, et al. Graphene as a novel matrix for the analysis of small molecules by MALDI-TOF MS. Anal Chem. 2010; 82(14): 6208-6214.

68. Wang Y, Li YM, Tang LH, et al. Application of graphene modified electrode for selective detection of dopamine. Electrochem Commun. 2009; 11(4): 889-892.

69. Chang HX, Tang LH, Wang Y, et al. Graphene fluorescence resonance energy transfer aptasensor for the thrombin detection. Anal Chem. 2010; 82(6): 2341-2346.

70. He QY, Sudibya HG, Yin ZY, et al. Centimeter-long and large-scale micropatterns of reduced graphene oxide films: fabrication and sensing applications. ACS Nano. 2010; 4(6):3201-3208.

71. Zeng Q, Cheng JS, Tang LH, et al. Self-assembled graphene-enzyme hierarchical nanostructures for electrochemical biosensing. Adv Funct Mater. 2010; 20(19): 3366-3372.

72. Zhang Q, Wu SY, Zhang L, et al. Fabrication of polymeric ionic liquid/graphene nanocomposite for glucose oxidase immobilization and direct electrochemistry. Biosensors \& Bioelectronics. 2011; 26(5): 2632-2637.

73. Wang Y, Zhang S, Du D, et al. Self assembly of acetylcholinesterase on a gold nanoparticles-graphene nanosheet hybrid for organophosphate pesticide detection using polyelectrolyte as a linker. J Mater Chem. 2011; 21(14): 5319-5325.

74. Zhang J, Dong XL, Chen JS, et al. Efficient Analysis of non-polar environmental contaminants by MALDI-TOF MS with graphene as matrix. J Am Soc Mass Spectr. 2011; 22(7): 1294-1298.

75. Wan Y, Wang Y, Wu JJ, et al. Graphene oxide sheet-mediated silver enhancement for application to electrochemical biosensors. Anal Chem. 2011; 83(3): 648-653.

76. Chen D, Tang LH, Li JH. Graphene-based materials in electrochemistry. Chem Soc Rev. 2010; 39(8): 3157-3180.

77. Peng $\mathrm{C}, \mathrm{Hu} \mathrm{WB}, \mathrm{Zhou} \mathrm{YT}$, et al. Intracellular imaging with a graphene-based fluorescent probe. Small. 2010; 6(15): 1686-1692.

78. Liu KP, Zhang JJ, Cheng FF, et al. Green and facile synthesis of highly biocompatible graphene nanosheets and its application for cellular imaging and drug delivery. J Mater Chem. 2011; 21(32): 12034-12040.

79. Pan DY, Zhang JC, Li Z, et al. Hydrothermal route for cutting graphene sheets into blue-luminescent graphene quantum dots. Advan Mater. 2010; 22(6): 734-738.

80. Eda G, Lin YY, Mattevi C, et al. Blue photoluminescence from Chemically Derived Graphene Oxide. Adv Mater. 2010; 22 (4): 505-509.

81. Mei QS, Zhang K, Guan GJ, et al. Highly efficient photoluminescent graphene oxide with tunable surface properties. Chem Commun. 2010; 46(39): 7319-7319.

82. Yan X, Cui X, Li LS. Synthesis of large, stable colloidal graphene quantum dots with tunable size. J Am Chem Soc. 2010; 132(17): 5944-5945.

83. Zhu SJ, Zhang JH, Qiao CY, et al. Strongly green-photoluminescent graphene quantum dots for bioimaging applications. Chem Commun. 2011; 47(24): 6858-6860.

84. Zhang LM, Xing YD, He NY, et al. Preparation of graphene quantum dots for bioimaging application. J of Nanosci Nanotechno; accepted.

85. Shen JH, Zhu YH, Chen C, et al. Facile preparation and upconversion luminescence of graphene quantum dots. Chem Commun. 2011; 47(9): 2580-2582.

86. Welsher K, Liu Z, Daranciang D, et al. Selective probing and imaging of cells with single walled carbon nanotubes as near-infrared fluorescent molecules. Nano Lett. 2008; 8(2): 586-590.

87. Sipkins DA, Cheresh DA, Kazemi MR, et al. Detection of tumor angiogenesis in vivo byav $\beta 3$-targeted magnetic resonance imaging. Nature Med. 1998; 4: 623-626.

88. Bonnemain B. Superparamagnetic Agents in magnetic resonance imaging: physicochemical characteristics and clinical applications a review. J Drug Targeting. 1998; 6(3): 167-174

89. Hu WB, Peng C, Luo WJ, et al. Graphene-based antibacterial paper. ACS Nano. 2010;4(7): 4317-4323.

90. Akhavan O, Ghaderi E. Toxicity of graphene and graphene oxide nanowalls against bacteria. ACS Nano. 2010; 4(10): 5731-5736.

91. Liu SB, Zeng TH, Hofmann M, et al. Antibacterial activity of graphite, graphite oxide, graphene oxide, and reduced graphene oxide: membrane and oxidative stress. ACS Nano. 2011; 5(9): 6971-6980.

92. Ryoo SR, Kim YK, Kim MH, et al. Behaviors of NIH-3T3 fibroblasts on graphene/carbon nanotubes: Proliferation, focal adhesion, and gene transfection studies. ACS Nano. 2010; 4(11): 6587-6598.

93. Nayak TR, Andersen H, Makam VS, et al. Graphene for controlled and accelerated osteogenic differentiation of human mesenchymal stem cells. ACS Nano. 2011; 5(6): 4670-4678.

94. Kalbacova M, Broz A, Kong J, et al. Graphene substrates promote adherence of human osteoblasts and mesenchymal stromal cells. Carbon. 2010; 48(15): 4323-4329. 
95. Li N, Zhang XM, Song $Q$, et al. The promotion of neurite sprouting and outgrowth of mouse hippocampal cells in culture by graphene substrates. Biomaterials. 2011; 32(35): 9374-9382

96. Zhang YB, Ali SF, Dervishi E, et al. Cytotoxicity effects of graphene and single-wall carbon nanotubes in neural phaeochromocytoma-derived PC12 cells. ACS Nano. 2010; 4(6): 3181-3186.

97. Chang YL, Yang ST, Liu JH, et al. In vitro toxicity evaluation of graphene oxide on A549 cells. Toxicol Lett. 2011; 200(3): 201-210.

98. Sasidharan A, Panchakarla LS, Chandran P, et al. Differential nano-bio interactions and toxicity effects of pristine versus functionalized graphene. Nanoscale. 2011; 3(6): 2461-2464.

99. Hu WB, Peng C, Lv M, et al. Protein corona-mediated mitigation of cytotoxicity of graphene oxide. ACS Nano. 2011;5(5): 3693-3700.

100.Yang K, Wan JM, Zhang S, et al. In vivo pharmacokinetics, long-term biodistribution, and toxicology of PEGylated graphene in mice. ACS Nano. 2011; 5(1): 516-522.

101.Zhang XY, Yin JL, Peng C, et al. Distribution and biocompatibility studies of graphene oxide in mice after intravenous administration. Carbon. 2011; 49(3): 986-995.

102.Liao KH, Lin YS, Macosko CW, et al. Cytotoxicity of graphene oxide and graphene in human erythrocytes and skin fibroblasts. ACS Appl Mater Interfaces. 2011; 3(7): 2607-2615.

\section{AUTHOR BIOGRAPHY}

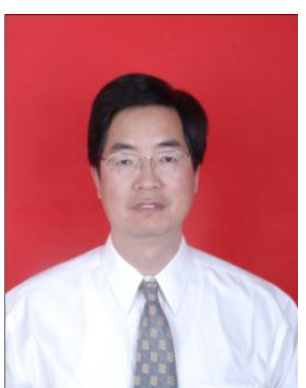

Dr. Zhijun Zhang is a professor of nanobiomedicine at Suzhou Institute of Nano-tech and Nano-bionics, Chinese Academy of Sciences. He has coauthored over 40 publications including Nature Materials, Nano Letters, Chem. Comm. and Small. He holds one US patent. The current research interests in Professor Zhang's group include: (1) Development of nanomaterials (graphene oxide, inorganic nanoparticles, and virus like particles)-based drug delivery system; (2) Synthesis of novel nanomaterials for biological detection and imaging; and (3) Bioeffects and safety of nanomaterials.

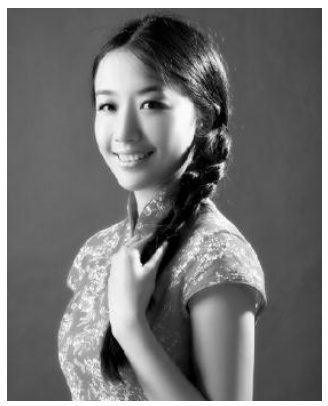

He Shen obtained her bachelor's degree from the School of Life Science, University of Science and Technology of China in 2009. She is currently a $\mathrm{Ph}$. D. student under the supervision of Prof. Zhijun Zhang. Her research is centered on development of graphene-based protein delivery systems.

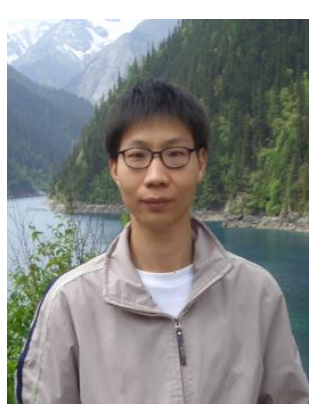

Dr. Liming Zhang earned his Ph. D. degree in Physical Chemistry from Suzhou Institute of Nano-tech and Nano-bionics, CAS, where he worked on development of graphene-based drug delivery systems with Prof. Zhijun Zhang. He is currently a staff scientist in Hunan Key Laboratory of Green Packaging and Application of Biological Nanotechnology, Hunan University of Technology, China.

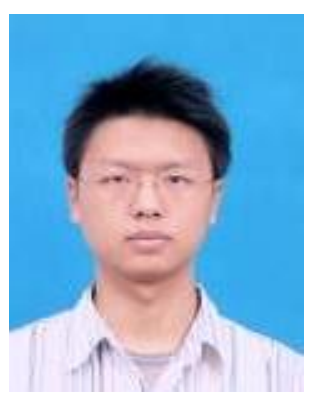

Dr. Min Liu obtained his doctor's degree from the College of Chemistry and Molecular Sciences, Wuhan University, China. He has coauthored over 10 publications including Biomaterials, Bioconjugate Chem., J. Mater. Chem., Macromol. Biosci., Polymer. Dr. Liu is a postdoctoral fellow in Prof. Zhijun Zhang's group. His current research focuses on using functionalized graphene and carbon nanotubes as gene and drug delivery systems. 